Dom. Cien., ISSN: 2477-8818

Vol. 3, núm. 3, julio, 2017, pp. 1373-1385

Necesidades de formación de los docentes de bachillerato

\title{
Necesidades de formación de los docentes de bachillerato
}

\section{Training needs of high school teachers}

\section{Necessidades de formação dos documentos de bachillerato}

\author{
Manuel W. Alvarado-Miles I \\ mwalvarado@uce.edu.ec \\ Francisco D. Sandoval-Ortiz ${ }^{\text {II }}$ \\ dantefran82@gmail.com \\ Edison A. Almachi-Montahuano III \\ María G. Figueroa-Arellano IV \\ gabicita30@yahoo.com \\ Marco V. Mier-Urbina V \\ eaalmachi@uce.edu.ec \\ mvmu41@gmail.com
}

Recibido: 30 de enero de 2017 * Corregido: 20 de febrero de $2017 *$ Aceptado: 20 junio de 2017

I. Magister en Gerencia y Liderazgo Educacional; Licenciado en Ciencias de la Educación Mención Ingles; Tecnólogo En Administración de Empresas; Técnico Superior en Administración de Empresas; Universidad Central del Ecuador, Quito, Ecuador.

II. Magister en Docencia Universitaria y Administración Educativa; Licenciado en Ciencias de la Educación Mención Ingles; Universidad Central del Ecuador, Quito, Ecuador.

III. Magister en Linguistica Aplicada a la Enseñanza Bilingue Español-Inglés; Licenciado en Ciencias de la Educación Mención Ingles; Técnico Superior en Análisis de Sistemas; Tecnólogo en Sistemas de Automatización; Universidad Central del Ecuador, Quito, Ecuador.

IV. Master of Science in Curriculum and Instruction, English as a Second Language; Ingeniera en Gestión Turística y Medio Ambiente; Guía de Turismo; Universidad Central del Ecuador, Quito, Ecuador.

v. Magister en Linguistica Aplicada a la enseñanza Bilingue Español-Inglés; Magister en Gerencia y Liderazgo Educacional; Licenciado en Ciencias de la Educación mención Ingles; Universidad Central del Ecuador, Quito, Ecuador. 


\section{Resumen}

El presente trabajo tuvo como objetivo general analizar las necesidades de formación de los docentes de bachillerato en el período lectivo 2012-2013, en este caso de los docentes del Colegio Particular Mixto Interandino de la ciudad de Quito.

El instrumento que se utilizó para la recolección de datos fue el cuestionario proporcionado por el Departamento de Ciencias de la Educación de la Universidad Técnica Particular de Loja.

Luego del análisis de los cuestionarios, se concluye que el principal obstáculo en la capacitación de los docentes de bachillerato del Colegio Interandino es la falta de apoyo de la institución, ya que no motiva a sus docentes a mantenerse capacitados y actualizados en la materia que cada uno tiene a su cargo. También se detectó falencias en el aspecto pedagógico, así como en la incorporación de las nuevas tecnologías al proceso de aprendizaje.

Para suplir esas deficiencias de los docentes se diseñó un curso de formación que trata sobre la creación y desarrollo de un entorno virtual de enseñanza-aprendizaje basado en las herramientas de libre uso asociadas a la WEB 2.0., para de esta manera mejorar los procesos de enseñanzaaprendizaje del colegio.

Palabras clave: Necesidades de formación; cuestionario; capacitación. 


\section{Abstract}

The aim of the current research was to analyze the training needs of high school teachers in the educational institutions nationwide, in the school term 2012-2013. The current study was carried out in the "Interandino" coeducational private high school, located in the city of Quito, in order to determine the training needs of teachers working at that institution.

The instrument used for data collection was the questionnaire provided by the Department of Science and Education of the "Universidad Técnica Particular de Loja". After analyzing the information from the questionnaires, the main conclusion is that the greatest obstacle in the training Interandino high school teachers is the lack of support and motivation by the institution in order to encourage teachers to stay trained and updated. In addition some knowledge gaps were detected in the pedagogical aspect as well as the slow incorporation of new technologies into the learning process.

As a result of the current study findings, a training program was designed for Interandino high school teachers.

Key words: Training needs; questionnaire; training program. 


\section{Introducción.}

El docente es un factor clave en el proceso de enseñanza-aprendizaje en todos los niveles, ya sea de educación inicial, educación básica, educación secundaria o educación superior. Desde luego las universidades son también actoras generadoras de cambio, y la Universidad Técnica Particular de Loja no puede ser la excepción, consciente de lo trascendentes que pueden ser sus aportes a la sociedad ecuatoriana, propuso a quienes cursan la maestría de Gerencia y Liderazgo Educacional, realizar la investigación titulada: Diagnóstico de necesidades de formación de los docentes de bachillerato. (Arenas Castellanos \& Fernández de Juan, 2009) (ANUIES, 2000)

Los resultados del presente trabajo revisten gran importancia ya que sirven de referente para mejorar y optimizar la gestión pedagógica de los docentes, pues se puede trabajar sobre aquellas debilidades o falencias que se encuentran en el ámbito de la formación y capacitación docente, lo que sin duda mejorará el desempeño docente y el proceso de aprendizaje de los estudiantes de nuestro país, cuyos colegios y maestros sirvieron como medio para esta investigación. (Rueda Beltrán, 2010) (López Bonilla \& Tinajero Villavicencio, 2009) (Francesc \& Puig, 1998)

El tema de la formación docente aporta elementos positivos no sólo para el sector educativo, sino para la sociedad en su conjunto por la sencilla razón que vivimos en la sociedad del conocimiento. El conocimiento está en constante movimiento y transformación; no obstante, el conocimiento también está sujeto a una prematura obsolescencia, lo que obliga a todos los profesionales a estar en un proceso de formación continua, caso contrario la o el profesional corre el riesgo de rezagarse en su crecimiento profesional. (García Aróstegui, 2008) (Torres del Castillo, 1998) 
La labor investigativa del presente trabajo condujo a indagar referentes teóricos en materia de necesidades de formación, análisis organizacional, análisis de la persona y análisis de la tarea educativa; todo ello conducente a construir el marco teórico que sirve de base para el presente trabajo académico.

La investigación fue factible a través de la aplicación de cuestionarios a los docentes del Colegio Particular Mixto Interandino, cuestionarios que fueron elaborados y facilitados por la Universidad Técnica Particular de Loja. Es importante mencionar que se contó con la total colaboración de los docentes del colegio antes mencionado, lo que contribuyó significativamente a su exitosa realización.

El objetivo de la presente investigación determinar las necesidades de formación de los docentes de bachillerato.

\section{Metodología.}

Diseño de la investigación.

El presente trabajo es de tipo descriptivo y exploratorio y tiene como objetivo principal determinar las necesidades de formación de los docentes de bachillerato, por lo que se debe analizar los datos obtenidos a través de los cuestionarios para tener una validez estadística de los resultados a través de la aplicación de la estadística descriptiva. Tomando en cuenta que se pudo aplicar el cuestionario al total de la población de docentes, objeto de la presente investigación es factible tener datos de carácter veraz y fidedigno que asegurará un resultado certero, además del hecho de aplicar variables discretas de orden cuantitativo y cualitativo. 
Métodos de investigación.

Los métodos aplicados en la presente investigación están basados en un enfoque investigativo cualitativo y cuantitativo, además de descriptivo y exploratorio.

Es cualitativo porque permite explicar y diagnosticar la capacitación docente en el Colegio Particular Mixto Interandino para determinar las necesidades de formación de los docentes de dicho colegio, así como para implementar alternativas de capacitación docente en la institución.

Es cuantitativo ya que a través de los valores obtenidos en las encuestas se pueden establecer porcentajes en el diagnóstico de necesidades de formación docente y sobre la base de los datos de las encuestas hacer un análisis comparativo.

Técnicas e instrumentos de investigación

Técnicas de investigación.

Las técnicas e instrumentos empleados en este trabajo simplifican los procesos de comprensión y síntesis de la información referente al diagnóstico de las necesidades de formación docente de bachillerato.

Las técnicas utilizadas en la presente investigación fueron:

La lectura: como método que permite el análisis pormenorizado de la información con el fin de seleccionar conceptos y referencias que aporten significativamente a la investigación.

Mapas conceptuales y organizadores gráficos: como métodos que permiten la comprensión y estructuración sintetizada de los aportes teóricos utilizados en la investigación. 
La encuesta: método que permite el acercamiento directo con la información y los sujetos de investigación, mediante interrogantes concretas, que abordan la problemática motivo de este estudio.

\section{Instrumentos de investigación.}

Cuestionario de necesidades de formación docente de bachillerato que fue proporcionado por el Departamento de Ciencias de la Educación y la coordinación de la titulación de la maestría en Gerencia y Liderazgo Educacional de la Universidad Técnica Particular de Loja (Anexo 2), el mismo que abarca los siguientes aspectos relativos a la formación docente: formación docente, cursos y capacitaciones, aspectos sobre la institución educativa donde laboran y lo relacionado a su práctica pedagógica.

Sistematización de los datos: para la tabulación de los datos se utilizó la plantilla Excel (xls.) y sus utilitarios compatibles con los sistemas operativos vigentes.

\section{Procedimiento.}

La presente investigación adquirió el siguiente modelo de proceso y cronología:

El primer paso fue determinar los posibles colegios donde podría llevarse a cabo la presente investigación, tomando en cuenta la factibilidad logística por parte del investigador, se procedió a llevar a cabo una breve entrevista con las autoridades de los potenciales planteles, teniendo la mejor acogida y apertura para llevar a cabo la investigación en el Colegio Particular Mixto Interandino, donde su Rectora otorgó la autorización respectiva. 
Luego se procedió a la aplicación de las encuestas en una fecha que fue acordada con una semana de anticipación, en donde se procedió a explicar a los diez docentes del colegio los aspectos relativos a la capacitación docente que contemplaba el instrumento de las encuestas. Los docentes fueron cooperativos y receptivos al instrumento de investigación.

Posteriormente se realizó la codificación de las encuestas llenadas por los docentes del colegio, a través de la elaboración de las correspondientes tablas estadísticas y una representación gráfica de cada tabla para una mejor compresión de los resultados obtenidos.

\section{Resultados.}

\section{Su titulación tiene relación con: Ámbito Educativo.}

En lo relacionado específicamente a necesidades formativas de los docentes se obtienen los siguientes resultados, el $80 \%$ de los docentes tienen el título de licenciatura en ciencias de la educación y el $20 \%$ restante no contesta al requerimiento. Tomando en cuenta las políticas actuales de educación en nuestro país y el deseo del actual gobierno de mejorar sustancialmente la educación es importante que los docentes en ejercicio posean una formación especializada en el campo educativo, caso contrario deberían buscar los medios para obtener esa experticia en educación.

\section{Su titulación tiene relación con: Otras profesiones.}

Sólo un $10 \%$ de los docentes tiene titulación en otra profesión que no es la docencia, mientras que el 90\% restante tiene su titulación en el campo de la educación. En este caso en concreto el docente tiene la profesión de abogado, y tomando en cuenta esa puntualización es de esperarse que el profesional en abogacía adquiera los conocimientos necesarios en pedagogía para transmitir de mejor manera su conocimiento. 


\section{Si posee titulación de postgrado, ésta tiene relación con: El Ámbito Educativo}

Ninguno de los docentes de la institución posee titulación de cuarto nivel ya sea en el ámbito educativo u otros ámbitos. Es altamente beneficioso para una institución educativa el contar con personal calificado profesionalmente y académicamente, pues esto permite elevar los estándares de calidad y eficiencia. Además el hecho de que existan profesionales de tercer nivel en la institución, motiva al resto de docentes a también buscar la capacitación y la formación de cuarto nivel.

\section{Les resulta atractivo seguir un programa de formación para obtener una titulación de cuarto nivel.}

Ante la inquietud de seguir la titulación de cuarto nivel, al 70\% de los docentes del Colegio Interandino les resulta atractivo el acceder a un programa de formación para obtener una titulación de cuarto nivel (tabla $\mathrm{N}^{\circ} 7$ ), sin embargo al 10\% no le resulta atractiva la propuesta y el $20 \%$ no responde a la inquietud. Sería ideal que la institución educativa busque una manera de incentivar al 70\% de docentes que sí están interesados en cursar la titulación de cuarto nivel, ya sea a través de una contribución económica así como de dar facilidades en cuanto a horarios de trabajo, ya que a futuro la institución podría ser la directamente beneficiaria de esa decisión.

\section{Cuáles son los obstáculos que se presentan para que usted no se capacite.}

Los obstáculos que tienen los docentes para acceder a la capacitación son primordialmente el $22.73 \%$ altos costos de los cursos o capacitaciones y falta de información. El $18.18 \%$ aduce a la falta de tiempo, y a la falta de apoyo por parte de las 
autoridades de la institución. El 13.64 aduce falta de temas acorde a su preferencia y el $4.55 \%$ restante no está interesado en la capacitación profesional.

Considerando en primer lugar el obstáculo que tiene mayor peso que es aquel de los altos costos de los cursos o capacitaciones, una forma de contrarrestar este limitante podría ser que la institución contribuya con un porcentaje de la misma y el docente no pague el valor en su totalidad. Por otro lado la falta de información respecto a capacitaciones que aducen los docentes, una forma de solucionar ese factor es el buscar convenios ya sea a través del Ministerio de Educación del Ecuador o a través de instituciones que brinden capacitación en las distintas áreas de conocimiento, siendo el colegio el vínculo que transmita a sus docentes esa información.

\section{Cuáles son los motivos por los que usted asiste a capacitaciones.}

Los motivos que llevan a los docentes a capacitarse, en primera instancia el $33.33 \%$ lo hace por la relación del curso con la actividad docente y porque les gusta la capacitación. El 16.66\% se capacita porque favorece a su ascenso profesional. El 8.33\% se capacita por el prestigio del ponente y por la facilidad de horarios.

Para que la formación consiga los objetivos pretendidos es imprescindible considerar la formación como un proceso de comunicación en el que tiene tanta relevancia el "cómo" se desarrolla el programa, como el "qué" o contenido de los temas del mismo. De igual manera es importante delimitar los objetivos que se pretende alcanzar con el curso, por lo que los objetivos deben ser definidos, alcanzables y evaluados antes, durante y después del proceso de formación. 
Un elemento extremadamente positivo es que al 33.33\% de los docentes les gusta capacitarse, ese es un factor que la institución debe explotar y contagiar al resto del personal docente de esa predisposición a la formación docente.

\section{Cuáles considera Ud. son los motivos por los que se imparten los cursos/capacitaciones.}

De acuerdo a la concepción de los docentes encuestados los motivos por los que se imparten los cursos o capacitaciones, son en un $29.17 \%$ debido a actualización de leyes y reglamentos, el $20.83 \%$ por requerimientos personales, el $25 \%$ por aparición de nuevas tecnologías, y el $12.5 \%$ por falta de cualificación profesional y necesidades de capacitación continua y permanente.

Se podría interpretar que al referirse a actualización de leyes y reglamentos, los docentes buscan estar informados en cuanto a la normativa legal que rige su actividad, así como saber sus derechos y obligaciones. El segundo motivo considerado para los cursos o capacitaciones por parte de los docentes es la aparición de nuevas tecnologías, lo que indica su interés por incorporarlas a su proceso de enseñanza.

\section{Conclusiones.}

Se presentan las conclusiones obtenidas en el presente trabajo sobre la base del cuestionario provisto por la Universidad Técnica Particular de Loja, el cual fue aplicado a los docentes del Colegio Particular Mixto Interandino en el período lectivo 2012-2013, y de cuyo análisis se obtuvieron las siguientes conclusiones:

1) Una vez aplicado el cuestionario de la UTPL y el posterior análisis de los mismos se pudo diagnosticar y determinar que existen necesidades de formación en los docentes de 
bachillerato del Colegio Particular Mixto Interandino, fundamentalmente debido a que la institución no propicia, ni apoya la capacitación docente que supla las necesidades formativas.

2) Un factor importante que limita a los docentes a capacitarse son los altos costos de los cursos de formación en el mercado, ya que aquellos que han logrado capacitarse lo han hecho por cuenta propia.

3) La totalidad de los docentes del Colegio Interandino manifiestan que es importante para ellos el seguirse capacitando en temas educativos, situación que debe ser percibida como una oportunidad por la institución y aprovechar esa motivación intrínseca por mejorar su actividad docente.

4) Ninguno de los docentes del Colegio Interandino posee título de cuarto nivel que corresponde a la titulación de maestría. No obstante el que no sea obligatorio el poseer un título de cuarto nivel para ejercer la docencia en colegios, al ochenta por ciento de los docentes de la institución les gustaría obtener una maestría.

5) Una vez que se determinó que los docentes tienen falencias y a la vez interés por preparase en la temática de nuevas tecnologías aplicadas a la educación, se diseñó un curso de formación para los docentes de bachillerato del Colegio Particular Mixto Interandino.

\section{Bibliografía.}

ANUIES. (2000). Evaluación del desempeño del personal académico: Análisis y propuesta metodológica básica. México: Asociación Nacional de Universidades e Instituciones de Educación Superior.

Arenas Castellanos, M., \& Fernández de Juan, T. (2009). Formación pedagógica docente y desempeño académico de alumnos en la facultad de Ciencias Administrativas de la UABC. Revista de la educación superior, 38(150), 7-18. 
Francesc, P., \& Puig, I. (1998). Las reformas educativas. Una perspectiva política y comparada. Buenos Aires: Paidós.

García Aróstegui, I. (2008). Competencias comunicativas del maestro en fomración. Granada: Universidad de Granada.

López Bonilla, G., \& Tinajero Villavicencio, G. (2009). Los docentes ante la reforma del bachillerato. Revista Mexicana de Investigación Educativa, 14(43), 1191-1218.

Rueda Beltrán, M. (2010). La docencia, factor clave en el cumplimiento de las metas educativas. Perfiles educativos, 32(129), 3-6.

Torres del Castillo, R. (1998). Nuevo papel docente. ¿Qué modelo de formación y para qué modelo educativo? Perfiles Educativos, 82(dic), 6-12. 